\title{
First-principles calculations of spin-dependent conductance of graphene flakes
}

\author{
H. Şahin ${ }^{1}$ and R. T. Senger ${ }^{2}$,* \\ ${ }^{1}$ UNAM-Institute of Materials Science and Nanotechnology, Bilkent University, 06800 Ankara, Turkey \\ ${ }^{2}$ Department of Physics, Bilkent University, 06800 Ankara, Turkey
}

(Received 20 July 2008; revised manuscript received 17 October 2008; published 18 November 2008)

\begin{abstract}
Using $a b$ initio density-functional theory and quantum transport calculations based on nonequilibrium Green's function formalism we study structural, electronic, and transport properties of hydrogen-terminated short graphene nanoribbons (graphene flakes) and their functionalization with vanadium atoms. Rectangular graphene flakes are stable, having geometric and electronic structures quite similar to that of extended graphene nanoribbons. We show that a spin-polarized current can be produced by pure hydrogenated rectangular graphene flakes by exploiting the spatially separated edge states of the flake using asymmetric nonmagnetic contacts. Functionalization of the graphene flake with magnetic adatoms such as vanadium also leads to spin-polarized currents even with symmetric contacts. We observe and discuss sharp discontinuities in the transmission spectra which arise from Fano resonances of localized states in the flake.
\end{abstract}

DOI: 10.1103/PhysRevB.78.205423

PACS number(s): 73.63. $-\mathrm{b}, 72.25 .-\mathrm{b}, 75.75 .+\mathrm{a}$

\section{INTRODUCTION}

In the last two decades various forms of carbon nanostructures, namely, buckyballs, carbon nanotubes, and lately graphene, have attracted a great deal of interest due to their novel fundamental properties and possible applications in electronics. With the developments in preparation and synthesis techniques carbon-based nanostructures have emerged as one of the most promising materials for nonsilicon electronics.

The fabrication of graphene sheets ${ }^{1}$ and observation of their unusual properties such as a half-integer quantum-Hall effect have attracted much interest in electronic transport properties of this type of two-dimensional graphitic materials. Observed gapless energy spectrum and high mobility electron transport ${ }^{2-4}$ are the most remarkable features of graphene. It was shown by tight-binding calculations considering the $\pi$ bands that in the electronic energy dispersion of graphene, energy is linearly dependent on the wave vector around the Fermi level ${ }^{5}$ which makes it a unique material.

In recent experimental studies graphene nanoribbons (GNRs) with narrow widths $(10-70 \mathrm{~nm})$ have been realized. ${ }^{6,7} \mathrm{Li}$ et al. ${ }^{7}$ reported the producing ultranarrow ribbons with widths down to a few nanometers. In addition to high carrier mobilities that are higher than those in commercial silicon wafers, existence of width-dependent energy band gaps makes the graphene nanoribbons a potentially useful structure for various applications. The width dependence of the band gap and transport properties in quasione-dimensional narrow GNRs have been studied theoretically. ${ }^{8-17}$

Graphene nanoribbons having $r$ zigzag rows ( $r$-ZGNRs) are predicted as semiconductors having a narrowing band gap with the increasing width of the ribbon. Armchair-edged graphene nanoribbons (AGNRs) are also semiconducting with direct band gaps. ${ }^{10}$ Density-functional theory (DFT) calculations predict a high density of states around the valence- and conduction-band edges of ZGNRs, which derive from the states that are localized at the edges of the ribbon, and lead to nonzero magnetic moments on the carbon atoms. The existence of such peculiar edge states in graphitic materials had been discussed in some earlier works. ${ }^{18-23} \mathrm{It}$ has been established that the ground state of a ZGNR is antiferromagnetic with ferromagnetically ordered spin polarization along the edges. ${ }^{9}$ Due to the fact that the states near the Fermi level are derived from the edge states and their linear combinations, external fields have more significant effects on the edge states. The nature of the interactions between the magnetized edges of ZGNR was studied, concluding that the main contribution to the local moments comes from dangling bonds, and their interaction is determined by tails of the edge-localized $\pi$ states. $^{9}$ Due to the edge effects, graphene ribbons show different one-dimensional transport properties from those of carbon nanotubes.

Since GNRs have long spin-correlation lengths and good ballistic transport characteristics they can be considered as a promising active material of spintronic devices. ${ }^{24-27}$ In particular ZGNRs, known to have large spin polarizations at the opposite edges of the ribbon, may be utilized to create spindependent effects such as spin-polarized currents without the need of ferromagnetic electrodes or other magnetic entities.

Hydrogen termination of the edge atoms by forming strong $\sigma$ bonds would be important for the structural and electronic stability of the graphene ribbon. Both firstprinciples and tight-binding calculations showed that the termination of edges with hydrogen atoms removes the electronic states related to the dangling bonds. ${ }^{28}$ However, there are no qualitative changes in the electronic structure and the magnetic order of the ZGNRs with hydrogen atom termination, ${ }^{16}$ except for a narrowing of the band gap as we calculated.

Modification of electronic structure by impurities, adatoms, and external fields is another potential of graphene and graphitic structures for applications in nanoelectronics. Effects on electronic properties and magnetic behavior of graphene by the adsorption of foreign atoms have been considered in some previous works. ${ }^{29-36}$ Sizable gap opening by hydrogen adsorption to Stone-Wales defect sites of graphene has been reported. ${ }^{37}$ In the case of substitutional boron atom ${ }^{38,39}$ many of the electronic properties have been studied and it is suggested that GNRs may be used as spin filter 
devices. ${ }^{40}$ Based on the spin-polarized ground state of ZGNRs their possible application as a spin-valve device was proposed by using tight-binding ${ }^{41,42}$ and $\mathbf{k} \cdot \mathbf{p}$ calculations. ${ }^{43}$ It is also predicted by using first-principles calculations that the electric current flowing on the ribbon can be made completely spin polarized under in-plane homogeneous electric fields. ${ }^{44,45}$

In this study we consider electronic transport properties of short graphene nanoribbons (graphene flakes) and their functionalization with vanadium atoms. Electronic and magnetic properties of various types of nanometer-sized graphene flakes have been reported before. ${ }^{46-54}$ We restrict our considerations, however, to rectangular flakes only, which are finite segments of perfect GNRs. In particular we calculate spindependent transmission spectra of the flakes when the electrodes make partial contacts along the zigzag rows of the flakes. The geometrical asymmetry of the contacts leads to a polarization in the spin states of transmitted electrons. Depending on the contact geometry and the electrode thickness the spin polarization of the transmission shows various forms around the Fermi level, including perfect (100\%) polarization at certain energy ranges. Then we perform firstprinciples calculations to investigate the optimized geometry and electronic properties of the flakes with adsorbate vanadium atoms. We discuss the binding sites for single and double adsorbate atoms on the graphene flake. By using the relaxed geometry of adsorbate-flake system, we calculate their conductance spectrum by using model metallic electrodes. The adsorbate vanadium atoms introduce additional states around the Fermi level modifying the conductance spectrum of the flake by breaking the spin symmetry of the conduction electrodes even when the electrodes make uniform contacts with the flake. We also find traces of Fano resonances in the conductance spectrum of the graphene flakes arising from the coupling of the extended states of the quantum channel with the localized states of the flake, which acts like a scattering quantum dot. A local density-of-states (LDOS) analysis shows that the discontinuity features with sharp peaks and dips in the conductance spectra are associated with perfectly localized states of the structure in the electrode-flake-electrode geometry.

\section{CALCULATION METHODS}

We performed first-principles total-energy calculations to obtain electronic structure and equilibrium geometries of rectangular graphene flakes, with and without adatoms, based on the pseudopotential density-functional theory. ${ }^{55}$ The spin-dependent exchange-correlation potential is approximated within the generalized gradient approximation ${ }^{56}$ (GGA). The software package atomistix toolkit ${ }^{57}$ (ATK), which employs local numerical basis orbitals and nonequilibrium Green's function formalism to calculate quantum conductance in electrode-device-electrode geometry, has been used in all total-energy and transport calculations.

Geometry optimizations of graphene flakes have been done by relaxing all atomic positions in supercell geometries with sufficient vacuum spaces (minimum of $10 \AA$ ) to prevent the interactions with periodic images of the structure.

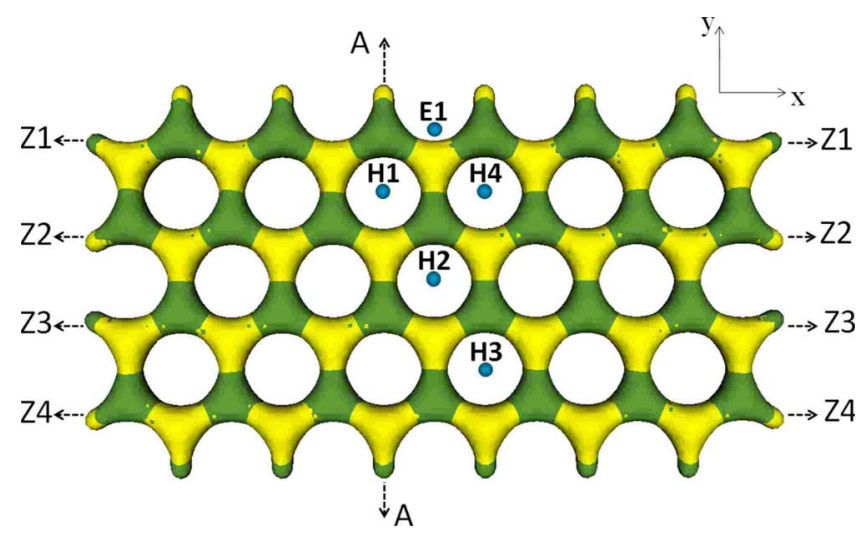

FIG. 1. (Color online) Geometry and spin-dependent charge density of the graphene flake cut from 4-ZGNR. The edges are saturated with hydrogen atoms. Green (dark) and yellow (light) regions denote the local majority-spin types of the charge density. Possible adsorption sites of adatoms are labeled as E1 and H1-H4. Electrodes (carbon chain or gold bar) are attached to the flake along directions $\mathrm{A}$ and $\mathrm{Z} 1-\mathrm{Z} 4$.

The criteria of convergence for total energy and HellmanFeynman forces were $10^{-4} \mathrm{eV}$ and $0.005 \mathrm{eV} / \AA$, respectively. The electrostatic potentials were determined on a realspace grid with a mesh cutoff energy of 150 Ry. Double-zeta double-polarized basis sets of local numerical orbitals were employed which give consistent results with previous calculations $^{58}$ that used plane-wave basis sets. In cases of extended nanoribbon calculations the Brillouin zone has been sampled with $(51,1,1)$ points within the MonkhorstPack $k$-point sampling scheme. ${ }^{59}$

The geometric structure of the graphene flake considered in this study is shown in Fig. 1. It is a finite segment of 4-ZGNR structure having four zigzag rows of carbon atoms along its axis. The sides along the cut direction have armchair shape. All edge carbon atoms have been saturated with hydrogen atoms for a better structural and electronic stability of the flake. Possible adsorption sites of adatoms have been denoted in the figure. The magnetic moments of the atoms and the structure as a whole have been calculated by using Mulliken analysis.

The spin-dependent transport properties of the graphene flake were calculated based on nonequilibrium Green's function formalism as implemented in ATK. Ballistic conductance spectrum of the flake is given within Landauer-Büttiker formalism as

$$
G(E)=G_{0} \sum_{\sigma} T_{\sigma}(E),
$$

where $G_{0}=e^{2} / h$ is the conductance quantum and $T_{\sigma}$ ( $\sigma=$ up, down) are the spin-dependent transmission spectra. For each spin state $\sigma$ the transmission probabilities are calculated as

$$
T_{\sigma}(E)=\operatorname{Tr}\left[\Gamma_{L} G^{r} \Gamma_{R} G^{a}\right]_{\sigma},
$$

where $G^{r}$ and $G^{a}$ are the retarded and advanced Green's functions and $\Gamma_{L}$ and $\Gamma_{R}$ are the contact broadening functions associated with the left and right electrodes, respectively. The broadening functions are anti-Hermitian components of 
the self-energy terms of the contacts, $\Sigma_{L}$ and $\Sigma_{R}$, which depend on the electrode's surface Green's function and the contact-molecule (graphene flake) bonding: $\Gamma_{L, R}=i\left(\Sigma_{L, R}\right.$ $\left.-\Sigma_{L, R}^{\dagger}\right) / 2$. The spatially separated spin states of the graphene flake are anticipated to break the spin symmetry in these conductance calculations when contacts to the electrodes are not symmetric at the armchair edges.

In order to exploit spin-dependent characteristics of the graphene flake the structure needs to be partially contacted with thin metallic electrodes. We used linear carbon atomic chains as model electrodes. Carbon atomic chains which are known to be metallic ${ }^{60}$ are expected to make reasonably good contact with the flake. The possible contact sites of the electrodes are denoted in Fig. 1. Semi-infinite carbon atomic chains have been rigidly attached to the flake at one or more sites along $\mathrm{A}$ and $\mathrm{Z} 1-\mathrm{Z} 4$ directions after removing the hydrogen atoms at the contact sites. Optimum electrode-flake distances were obtained by relaxation of the supercell consisting of the flake and four buffer carbon atoms per each carbon chain attached on both sides of the flakes.

As an alternative to carbon-chain electrodes we also considered gold electrodes to test the robustness of our conductance calculations. The gold electrodes were devised by taking a finite cross-section bar of atoms oriented in the [001] direction of fcc crystal. The semi-infinite gold bars have a unit cell of nine atoms in two layers of four and five atoms. We have added single gold atoms to the ends of the electrodes to get atomically sharp tips. Contact regions of the electrodes to the flake were geometrically optimized.

\section{RESULTS AND CONCLUSIONS}

For the generation of spin-polarized currents using a graphene flake, formation of spin-ordered edge-localized states found along the zigzag edges is the key mechanism. Therefore, we first calculate the ground-state magnetic ordering in ZGNR structures and compare it to that of the flake. In their ground state, all the carbon atoms of ZGNRs have antiparallel magnetic moments with their nearest neighbors, and their magnitudes rapidly decrease as we go away from the edges. For instance, according to the Mulliken population analysis moments of the atoms in the edge zigzag row of a 4-ZGNR alternate between $1.21 \mu_{B}$ and $-0.10 \mu_{B}$ per atom. In the second zigzag row the corresponding values are $0.19 \mu_{B}$ and $-0.07 \mu_{B}$. Relatively high magnetic moments are found on the atoms that are undercoordinated. Upon saturation of these carbons with hydrogen atoms the moments on the edge carbon atoms are reduced but the qualitative properties of the magnetic ordering of the ZGNRs persisted. For comparison, in the hydrogen-saturated 4-ZGNR the edge-row carbon atoms have alternating moments of $0.26 \mu_{B}$ and $-0.06 \mu_{B}$. In the second row the sequence of moments reduce to $0.07 \mu_{B}$ and $-0.04 \mu_{B}$. The hydrogen atoms too attain minute moments of about $0.01 \mu_{B}$, aligned antiparallel to those of the carbon atoms they are attached to. The spin-polarized edge structure of hydrogenated ZGNRs persists for wider ribbons, even with a slight increase in the moments of edge atoms. Hydrogenization also decreases the energy band gap of the $r$-ZGNRs. For instance, we calculated that the band-gap val- ues for $r=3,4,5$ reduce from $1.25,1.15,1.00$, to $0.70,0.65$, 0.60 in units of $\mathrm{eV}$, respectively.

Geometric and electronic structures of finite segments of ZGNRs, namely, rectangular graphene flakes, are quite similar to those of extended ribbons. Figure 1 shows the particular graphene flake $\left(\mathrm{C}_{52} \mathrm{H}_{20}\right)$ considered for the calculations in this study. Additional armchair edges of the flakes are also saturated with hydrogen atoms. Spin-unrestricted ground state of the flake corresponds to the antiferromagnetic spin coupling between the zigzag edges of the flake. The total energy of this state is $0.31 \mathrm{eV}$ lower than that of the ground state of a spin-restricted calculation, which is consistent with the calculations on extended ZGNRs. ${ }^{44}$ We find that away from the armchair edges of the flake the magnetic moments of the zigzag-edge carbon atoms are close to that of infinite ZGNR structure but tend to decrease toward the armchair edges. For instance, the moments of the six carbon atoms located at the uppermost zigzag edge of the flake shown in Fig. 1 are $0.09,0.21,0.29,0.29,0.21$, and 0.09 in units of $\mu_{B}$. The magnetic-moment profile of the carbon atoms rapidly converges to the ribbon values for longer flakes.

We calculate the spin-dependent ballistic conductance of the graphene flake when contacted with metallic electrodes. Since zero-bias conductance and transmission probability spectra are related by a simple multiplicative factor in Eq. (1), we frequently use these terms as equivalent quantities in the following discussions. By exploiting the spatial separation of spin states at the opposite zigzag edges of the flake we show that spin-polarized currents can be achieved without using ferromagnetic electrodes and magnetic entities. By making partial contacts to the armchair sides of the flake the spin symmetry in the coupling of the flake's electronic states to the electrodes is broken. Consequently the transmission spectrum of the electrons through the flake becomes dependent on their spin state. For a clear demonstration of these effects, stripped off complications that may arise from electrode-graphene interactions, we chose to use simple model electrodes. Carbon linear atomic chains are appealing in that respect, being metallic, making naturally good contact with graphene, and having atomically sharp tips.

In Fig. 2 we plot equilibrium transmission spectra of the bare and hydrogenated graphene flakes with single carbon atomic chain electrodes asymmetrically contacted to one of the edge zigzag rows of the flakes. For comparison, the spectrum of hydrogenated flake with more realistic gold electrodes is also presented. Presence of dangling bonds in the bare flake is reflected in the transmission spectrum as several sharp peaks about $0.7-1.0 \mathrm{eV}$ above the Fermi level. These peaks are removed after hydrogenization of the flake and are replaced by a single broad peak degenerate for both spin channels. We should note that within the chosen convention of labeling spin states the electrodes are contacted at the flake edge where up-spin states were localized. Therefore, the up-spin states around the Fermi-level couple more effectively with the electrodes leading to formation of a broader peak for up-spin channel in the differential conductance spectrum, and consequently the spin polarization of the current for small bias voltages is expected to reflect the flake's local majority-spin type of the contact side. With the gold electrodes, the basic features in the transmission spectrum 
remain unchanged except for the transmission peaks being narrower. The narrower peaks are due to weaker coupling of carbon atoms to gold electrodes.

The effect of electrode thickness and its contact location on the transmission spectrum is further demonstrated in Fig. 3 . When the carbon electrodes make a wider contact with the hydrogenated flake at Z1 and Z2 sites the spin asymmetry in the conductance spectrum is blurred with broader and overlapping peaks, but still the up-spin channel has a larger contribution to the conductance of the flake at energies around the Fermi level. In case of uniformly contacting electrodes (Z1-Z4) the transmission spectrum is identical for both spin channels as expected. Similarly, conductance of the flake when the electrodes are contacted along the armchair direction (contact site A in Fig. 1) is spin symmetric (not shown).

Another mechanism to break spin symmetry in the conductance of graphene flakes is to introduce magnetic impurities or adatoms. Here we present a systematic study of adsorption of vanadium atoms on graphene flake. Single atoms of several elements are adsorbed on carbon nanotube surfaces with relatively high binding energies and magnetic moments. ${ }^{58}$ Similar properties can be expected for their adsorption on graphene. Vanadium is a suitable element to consider due to its high binding energy and spin polarization. The ground-state electronic configuration of vanadium is $[\mathrm{Ar}] 4 s^{2} 3 d^{3}$, and it is a chemically active element with a magnetic moment of $3 \mu_{B}$.

We investigated the adsorption of single or double vanadium atoms on the graphene flake. Possible adsorption sites of vanadium atoms on the graphene flake are shown in Fig. 1. When there are two vanadium adatoms their locations relative to the flake are denoted by top (t) or bottom (b). Our results are summarized in Table I. Binding energies per adatom are calculated by using the expression $E_{b}=(E[\mathrm{G}]$ $+n E[\mathrm{~V}]-E[\mathrm{G}+n \mathrm{~V}]) / n$. Here, $n$ is the number of adsorbed $\mathrm{V}$ atoms and $E[\mathrm{G}]$ and $E[\mathrm{~V}]$ are the total energies of the graphene flake $\left(\mathrm{C}_{52} \mathrm{H}_{20}\right)$ and a free vanadium atom, respectively. Total energy of the relaxed structure when foreign atoms are adsorbed on graphene is denoted by $E[\mathrm{G}+n \mathrm{~V}]$. Like most of the $3 d$ transition-metal elements ( $\mathrm{Sc}, \mathrm{Ti}, \mathrm{Cr}$, $\mathrm{Mn}, \mathrm{Fe}, \mathrm{Co}$ ) vanadium atoms are adsorbed on hollow sites of two-dimensional graphene sheet. Due to the edge effects, however, on the graphene flake vanadium atoms are not adsorbed on the $\mathrm{H} 1, \mathrm{H} 3$, or $\mathrm{H} 4$ sites but rather they migrate to the E1 site after relaxation. We found that vanadium atom can be adsorbed also on the $\mathrm{H} 2$ site, but the binding energy is less than the one at the E1 site. H2-site adsorption is also off center; equilibrium position of the vanadium atom tends toward one of the hexagon sides.

Adsorbed vanadium atoms modify the spin-relaxed charge density of the flake; however the planar geometric structure of the graphene flake is quite robust to vanadium adsorption, the displacements of even the nearest-neighbor carbon atoms are less than 0.05 after geometry relaxation. As shown in Table I depending on the initial spin configurations of the flake and vanadium we identify two distinct magnetic states with close energies for each adsorption site of E1 and $\mathrm{H} 2$. In its ground state a vanadium atom adsorbed at the E1 site has a magnetic moment antiparallel with the moments of the edge carbon atoms as depicted in Fig. 4(a).

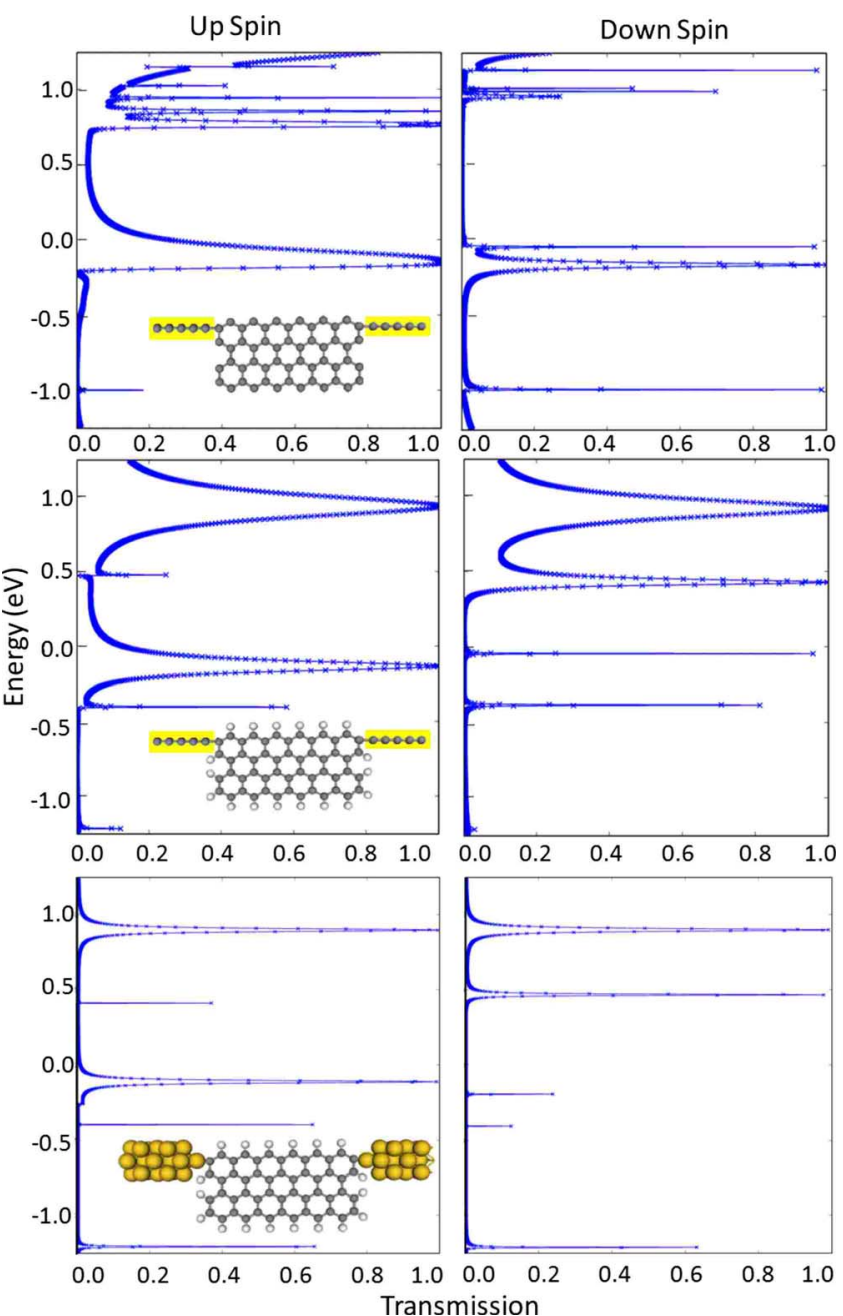

FIG. 2. (Color online) Spin-dependent transmission spectrum of the graphene flake without (top panels) and with (middle panels) hydrogen saturation of the edge atoms. The carbon linear-chain electrodes are connected to the Z1 sites (see Fig. 1). The spectra when the carbon-chain electrodes are replaced by gold leads are shown in the bottom panels. Fermi level is set to zero.

Binding of two adsorbate vanadium atoms on the same side of the flake is always stronger than that of being on different sides. For both single-sided and double-sided cases, two vanadium adatom configurations with antiparallel magnetic moment have larger binding energies than the cases with parallel magnetic moments.

By means of magnetic dipole-dipole interactions stronger bindings occur between the edge carbon atoms and vanadium adatom. Considerable change in the spin charge density of flake with vanadium adsorbate atoms (see Fig. 4) also modifies the conductance spectrum of the structure. In Fig. 5 it is seen that the adsorption of a vanadium atom at the E1 site with spin-down magnetic moment enhances the downspin transmission channel around the Fermi level. Spin polarization of the conductance spectrum is present even when the electrodes make uniform contacts with the flake.

In Fig. 6 we present an alternative view of the spin asymmetry in the conductance spectra by the plots of energydependent relative spin polarization of the transmission prob- 


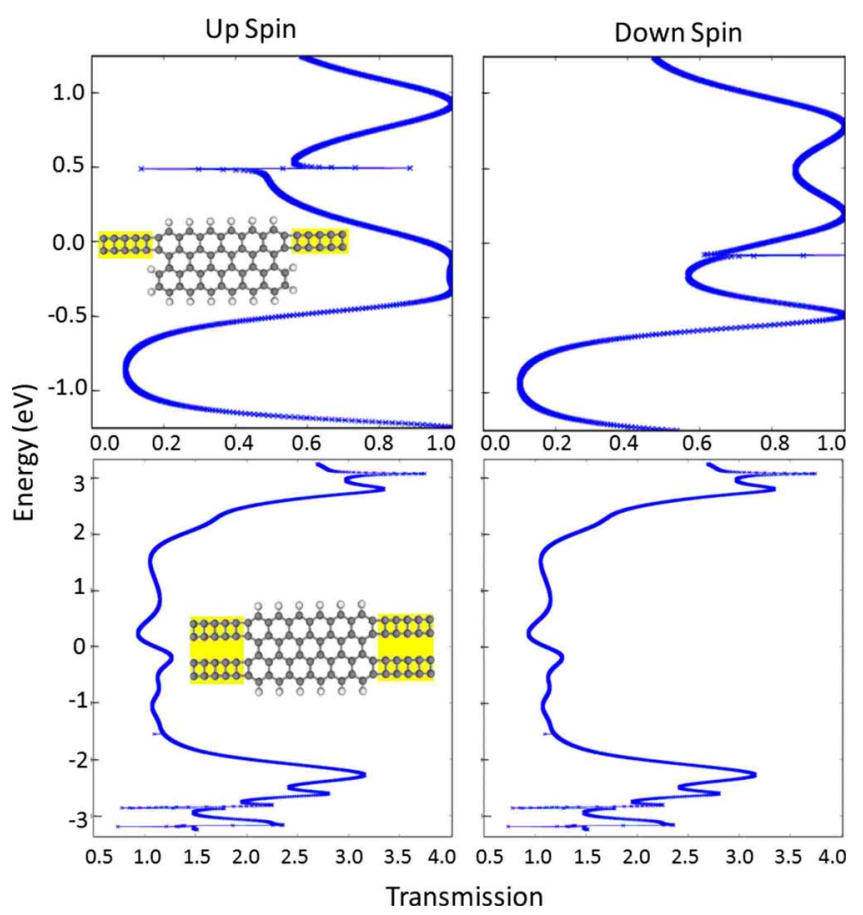

FIG. 3. (Color online) Spin-dependent transmission spectra of the hydrogen-saturated graphene flake when the electrodes make partial contact at the Z1 and Z2 sites (top panels) and when the contacts are uniform (bottom panels). Fermi level is set to zero.

abilities, $\quad\left(T_{\text {up }}-T_{\text {down }}\right) /\left(T_{\text {up }}+T_{\text {down }}\right)$, calculated for four different cases. Partial contacts of the electrodes and presence of magnetic adatoms both produce spin polarizations in the spectra. When the flake is contacted from the Z1 sites either with carbon chain or gold electrodes the polarization is almost $100 \%$ for an energy window of $\sim 0.8 \mathrm{eV}$ around the

TABLE I. Calculated properties of vanadium adatoms on the graphene flake. See Fig. 1 for the notation of adsorption sites. The magnetic order in the flake before the adsorption is such that the magnetic moments of the carbon atoms at the top edge are up $\left(\mu_{C}>0\right)$. In cases of double adsorbate atoms their locations relative to the flake have been denoted by top (t) or bottom (b). Magnetic moments of the vanadium adatoms $\left(\mu_{\mathrm{V}}\right)$ and the total moments of the structures $\mu_{\mathrm{tot}}$ are in units of Bohr magneton $\left(\mu_{B}\right) . E_{b}$ is the binding energy per vanadium adatom and $d_{\mathrm{G}-\mathrm{V}}$ is the equilibrium distance of the adatoms to the plane of graphene flake.

\begin{tabular}{lcrcc}
\hline \hline Adsorption site & $\mu_{\mathrm{V}}$ & $\mu_{\mathrm{tot}}$ & $\begin{array}{c}E_{b} \\
(\mathrm{eV})\end{array}$ & $\begin{array}{c}d_{\mathrm{G}-\mathrm{V}} \\
(\AA)\end{array}$ \\
\hline $\mathrm{E} 1\left(\mu_{\mathrm{V}}<0\right)$ & -3.8 & -4.9 & 1.59 & 1.94 \\
$\mathrm{E} 1\left(\mu_{\mathrm{V}}>0\right)$ & 3.8 & 3.0 & 1.58 & 1.94 \\
$\mathrm{H} 2\left(\mu_{\mathrm{V}}<0\right)$ & -3.9 & -4.3 & 1.01 & 1.86 \\
$\mathrm{H} 2\left(\mu_{\mathrm{V}}>0\right)$ & 3.9 & 4.4 & 1.02 & 1.87 \\
$\mathrm{H} 1-\mathrm{H} 2(\mathrm{t}-\mathrm{t})$ & $-2.5,2.0$ & 0.0 & 2.09 & $1.79,1.71$ \\
$\mathrm{H} 1-\mathrm{H} 3(\mathrm{t}-\mathrm{t})$ & $3.6,-3.6$ & 0.0 & 1.42 & $1.77,1.77$ \\
$\mathrm{H} 1-\mathrm{H} 4(\mathrm{t}-\mathrm{t})$ & $-1.9,1.8$ & 0.0 & 2.41 & $1.74,1.74$ \\
$\mathrm{H} 2-\mathrm{H} 2(\mathrm{t}-\mathrm{b})$ & $3.5,-3.5$ & 0.0 & 1.12 & $1.75,1.75$ \\
E1-E1 $(\mathrm{t}-\mathrm{b})$ & $3.7,-3.6$ & 0.9 & 1.28 & $1.98,1.96$ \\
\hline \hline
\end{tabular}
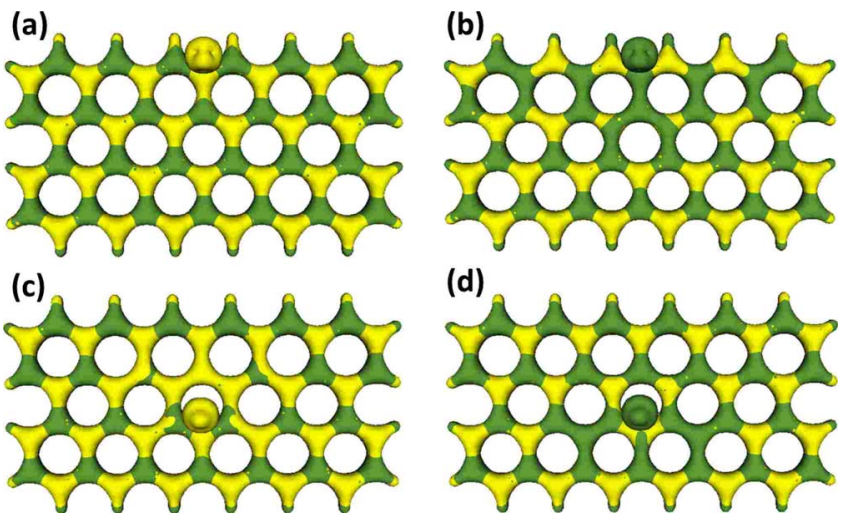

FIG. 4. (Color online) Majority-spin type of local charge density of the flake with single vanadium adatoms. The upper zigzag edge of the flake was spin-up polarized before the adsorption. The vanadium atom has a down magnetic moment in (a) and (c) and an up moment in (b) and (d). The lowest energy configuration for a single vanadium atom is shown in (a).

Fermi level. We also see that while uniformly contacted flake does not exhibit spin polarization, it can be generated by a single vanadium adatom.

Before we conclude, we would like to discuss several discontinuity features found in the transmission spectra, a clear example of which can be seen in top panels of Fig. 3. These are in the form of a pulsative variation with very narrow dips and adjacent peaks in the transmission function, reminiscent of a resonant effect. Similar resonances have been recently reported in the transmission spectra of carbon

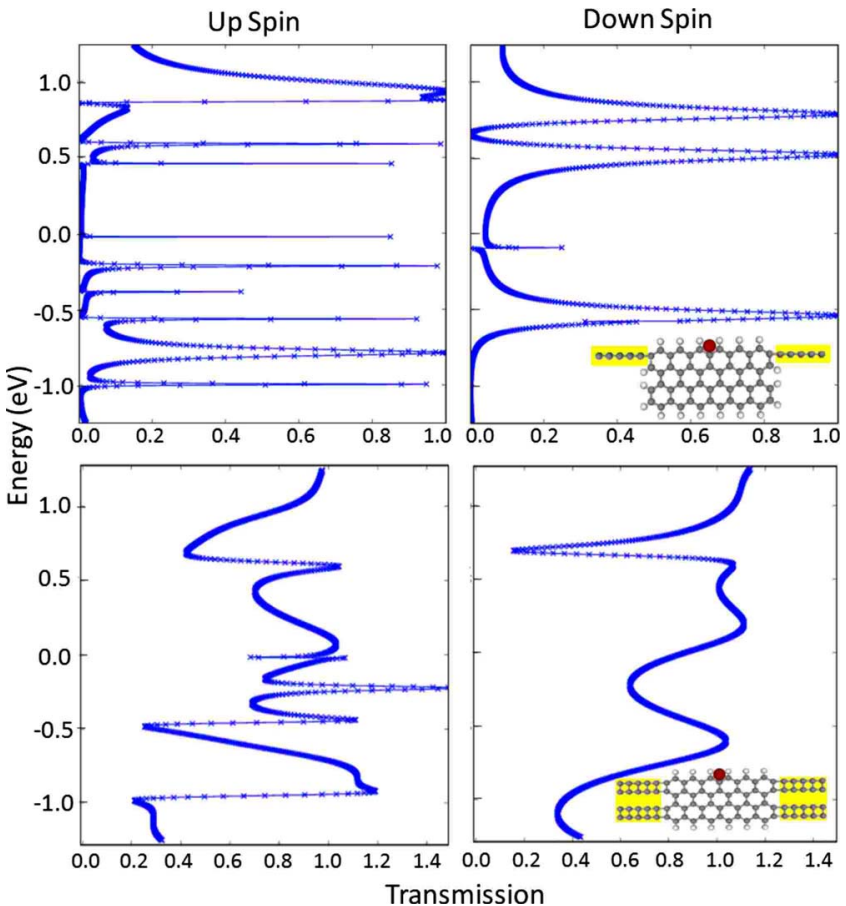

FIG. 5. (Color online) Spin-dependent transmission spectra of the hydrogen-saturated graphene flake with an adsorbed vanadium atom at the E1 site in its ground state. Top panels: the electrodes make partial contacts at the $\mathrm{Z} 1$ sites. Bottom panels: when the contacts are uniform. 

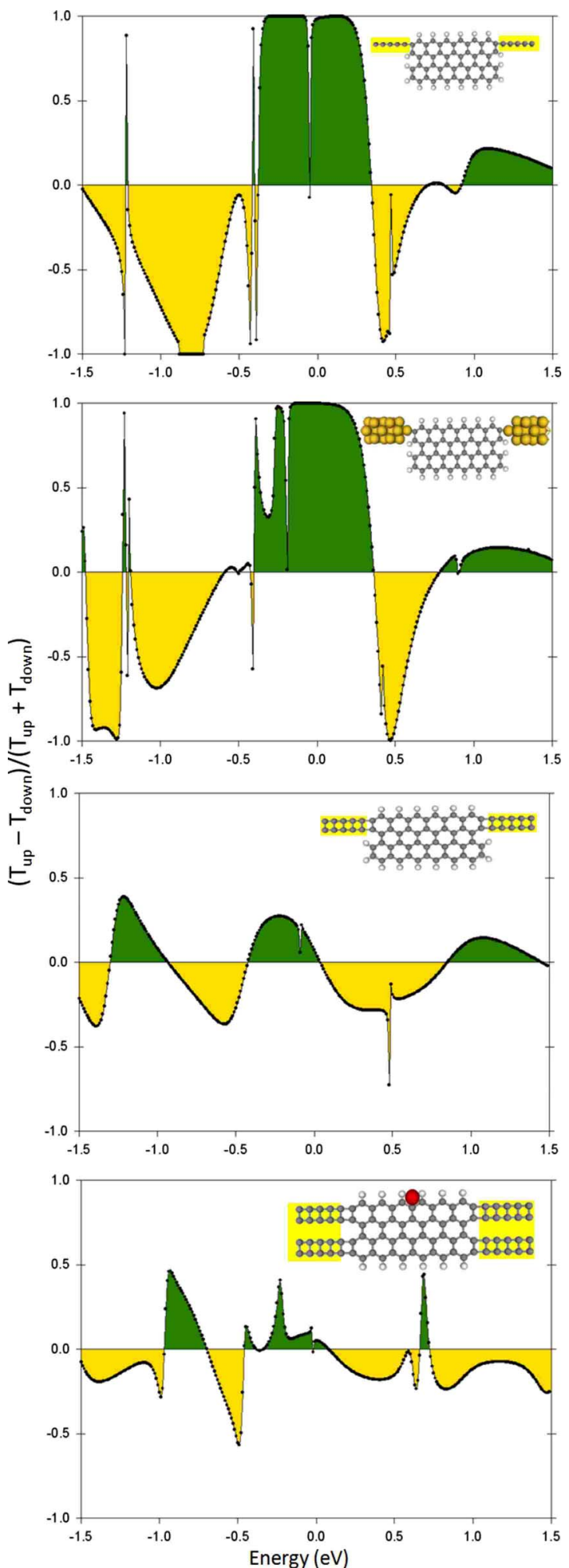

FIG. 6. (Color online) Spin polarization in the transmission spectra of the graphene flake. The type and contact geometries of the electrodes are given as insets. In the lowest panel there is a single vanadium adatom in its ground state.

nanotubes with a single iron or vanadium adsorbed atom on them. ${ }^{61}$ The underlying mechanism of these variations in the transmission function is the Fano resonances of localized
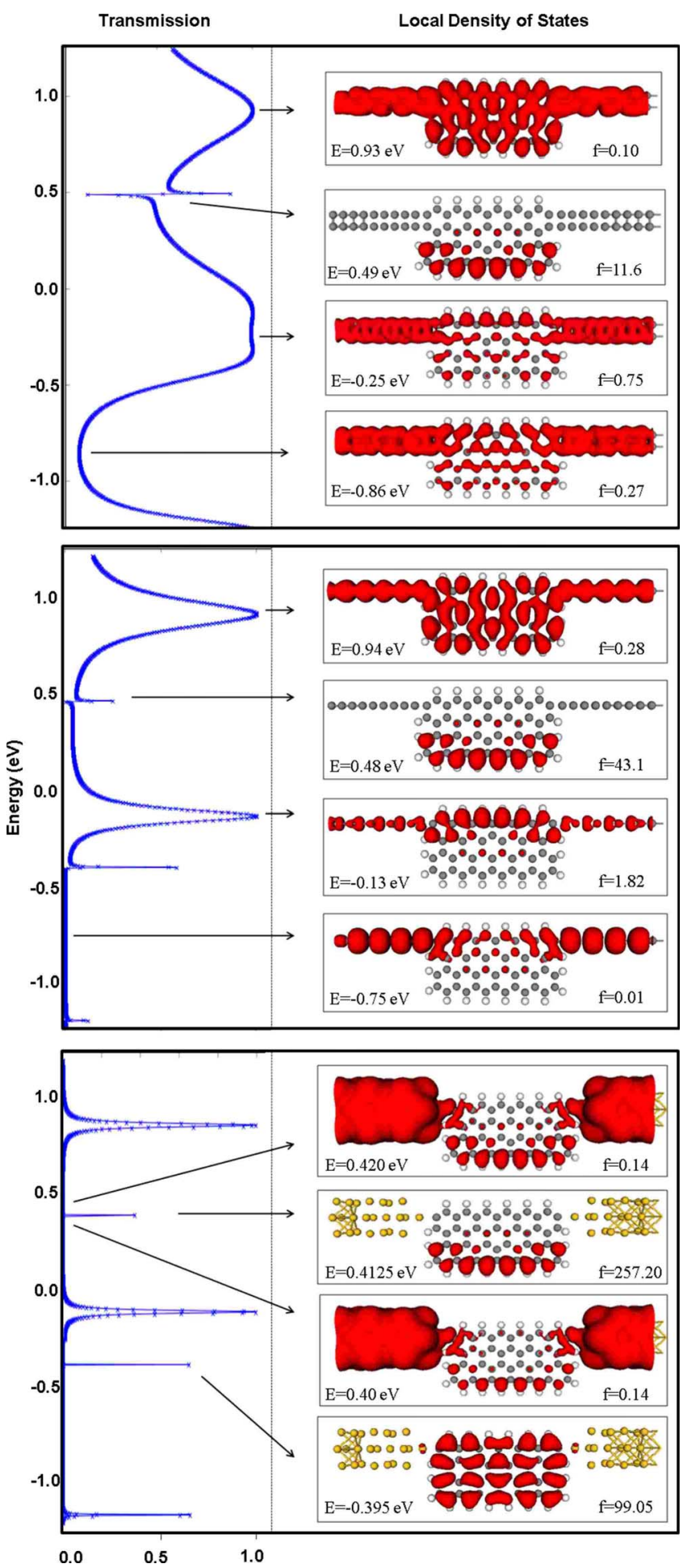

FIG. 7. (Color online) LDOS isosurfaces calculated for particular energy values of the up-spin transmission spectra of partially contacted graphene flake. The $f$ value next to each plot is the maximum value of LDOS obtained in units of states/ $\left(\AA^{3} \mathrm{eV}\right)$ and can be taken as a measure of relative magnitudes among the plots. The plotted isosurfaces correspond to LDOS values of $f \times 10^{-2}$.

states of the structure with extended band states of the electrodes with matching symmetry. The origin of the localized states contributing to the resonances can be either the local- 
ized $d$ states of the adatoms as in Ref. 61 and in the vanadium adatom parts of the present study or more generally they can be the localized states of a quantum dot coupled to the continuous states of a quantum channel. ${ }^{62}$ For instance, in formation of the resonances found in Fig. 3 the graphene flake acts like a scattering quantum dot coupled to the quantum channel of carbon-chain electrodes.

We infer about the character of these resonances by calculating LDOS of the structure at resonance and offresonance energies of the transmission spectra. In Fig. 7 we present LDOS isosurface plots for up-spin transmission spectra of three particular contact geometries. LDOS is perfectly confined at the flake region only at resonance energies. At off-resonance energies we always find contribution of extended states of the metallic electrodes.

In summary, we have performed an $a b$ initio study of spin-resolved conductance spectra of rectangular graphene flakes. We have shown that spin-polarized currents can be obtained by making the electrodes contact the flake partially along the zigzag-edge directions of the flake or by introducing transition-metal adatoms such as vanadium to alter the symmetry in spin-dependent scattering rates of the transmitted electrons. In the particular model system considered in this study ( $\mathrm{a} \mathrm{C}_{52} \mathrm{H}_{20}$ flake cut from 4-ZGNR) a $100 \%$ spin polarization in the conductance spectrum was achieved for both model carbon atomic chain and more realistic gold bar electrodes. Traces of Fano resonances have been found in the transmission functions. We believe that the qualitative features of producing spin-polarized currents in graphene flakes can be verified experimentally with flakes of larger sizes and with realistic electrodes. An understanding and realization of spin-dependent effects in graphitic structures are essential toward the development of graphene-based spintronic devices.

\section{ACKNOWLEDGMENTS}

This work was supported by TÜBITAK under Grant No. 106T597 and through TR-Grid e-Infrastructure Project. R.T.S. acknowledges financial support from Turkish Academy of Sciences (TÜBA) GEBİP program. *senger@fen.bilkent.edu.tr

${ }^{1}$ K. S. Novoselov, D. Jiang, F. Schedin, T. J. Booth, V. V. Khotkevich, S. V. Morozov, and A. K. Geim, Science 306, 666 (2004).

${ }^{2}$ K. S. Novoselov, A. K. Geim, S. V. Morozov, D. Jiang, M. I. Katsnelson, I. V. Grigorieva, S. V. Dubonos, and A. A. Firsov, Nature (London) 438, 197 (2005).

${ }^{3}$ Y. Zhang, Y.-W. Tan, H. L. Stormer, and Philip Kim, Nature (London) 438, 201 (2005).

${ }^{4}$ C. Berger, Z. Song, X. Li, X. Wu, N. Brown, C. Naud, D. Mayou, T. Li, J. Hass, A. N. Marchenkov, E. H. Conrad, P. N. First, and W. A. de Heer, Science 312, 1191 (2006).

${ }^{5}$ P. R. Wallace, Phys. Rev. 71, 622 (1947).

${ }^{6}$ M. Y. Han, B. Özyilmaz, Y. Zhang, and P. Kim, Phys. Rev. Lett. 98, 206805 (2007).

${ }^{7}$ X. Li, X. Wang, L. Zhang, S. Lee, and H. Dai, Science 319, 1229 (2008).

${ }^{8}$ Y. Miyamoto, K. Nakada, and M. Fujita, Phys. Rev. B 59, 9858 (1999).

${ }^{9}$ H. Lee, Y. W. Son, N. Park, S. Han, and J. Yu, Phys. Rev. B 72, 174431 (2005).

${ }^{10}$ Y. W. Son, M. L. Cohen, and S. G. Louie, Phys. Rev. Lett. 97, 216803 (2006).

${ }^{11}$ V. Barone, O. Hod, and G. E. Scuseria, Nano Lett. 6, 2748 (2006).

${ }^{12}$ K. I. Sasaki, S. Murakami, and R. Saito, J. Phys. Soc. Jpn. 75, 074713 (2006).

${ }^{13}$ D. A. Abanin, P. A. Lee, and L. S. Levitov, Phys. Rev. Lett. 96, 176803 (2006).

${ }^{14}$ D. A. Areshkin, D. Gunlycke, and C. T. White, Nano Lett. 7, 204 (2007).

${ }^{15}$ A. Cresti, G. Grosso, and G. P. Parravicini, Phys. Rev. B 76, 205433 (2007).

${ }^{16}$ K. N. Kudin, ACS Nano 2, 516 (2008).
${ }^{17}$ M. Ezawa, Phys. Rev. B 73, 045432 (2006).

${ }^{18}$ K. Kobayashi, Phys. Rev. B 48, 1757 (1993).

${ }^{19}$ M. Fujita, K. Wakabayashi, K. Nakada, and K. Kusakabe, J. Phys. Soc. Jpn. 65, 1920 (1996).

${ }^{20}$ K. Nakada, M. Fujita, G. Dresselhaus, and M. S. Dresselhaus, Phys. Rev. B 54, 17954 (1996).

${ }^{21}$ K. Wakabayashi, M. Sigrist, and M. Fujita, J. Phys. Soc. Jpn. 67, 2089 (1998).

${ }^{22}$ K. Nakada, M. Igami, and M. Fujita, J. Phys. Soc. Jpn. 67, 2388 (1998).

${ }^{23}$ K. Wakabayashi, M. Fujita, H. Ajiki, and M. Sigrist, Phys. Rev. B 59, 8271 (1999).

${ }^{24}$ P. Gambardella, A. Dallmeyer, K. Maiti, M. C. Malagoli, W. Eberhardt, K. Kern, and C. Carbone, Nature (London) 416, 301 (2002).

${ }^{25}$ J. Dorantes-Davila and G. M. Pastor, Phys. Rev. Lett. 81, 208 (1998).

${ }^{26}$ A. Vindigni, A. Rettori, M. G. Pini, C. Carbone, and P. Gambardella, Appl. Phys. A: Mater. Sci. Process. 82, 385 (2006).

${ }^{27}$ A. Delin, E. Tosatti, and R. Weht, Phys. Rev. Lett. 92, 057201 (2004).

${ }^{28}$ T. Kawai, Y. Miyamoto, O. Sugino, and Y. Koga, Phys. Rev. B 62, R16349 (2000).

${ }^{29}$ P. O. Lehtinen, A. S. Foster, A. Ayuela, A. Krasheninnikov, K. Nordlund, and R. M. Nieminen, Phys. Rev. Lett. 91, 017202 (2003).

${ }^{30}$ K. Nordlund, J. Keinonen, and T. Mattila, Phys. Rev. Lett. 77, 699 (1996).

${ }^{31}$ M. Heggie, B. R. Eggen, C. P. Ewels, P. Leary, S. Ali, G. Jungnickel, R. Jones, and P. R. Briddon, Proc.-Electrochem. Soc. 6, 60 (1998).

${ }^{32}$ Y. H. Lee, S. G. Kim, and D. Tomanek, Phys. Rev. Lett. 78, 2393 (1997).

${ }^{33}$ O. Hod, V. Barone, J. E. Peralta, and G. E. Scuseria, Nano Lett. 
7, 2295 (2007).

${ }^{34}$ D. Gunlycke, J. Li, J. W. Mintmire, and C. T. White, Appl. Phys. Lett. 91, 112108 (2007).

${ }^{35}$ E. J. Kan, Z. Y. Li, J. L. Yang, and J. G. Hou, J. Am. Chem. Soc. 130, 4224 (2008).

${ }^{36}$ H. Sevincli, M. Topsakal, E. Durgun, and S. Ciraci, Phys. Rev. B 77, 195434 (2008).

${ }^{37}$ E. J. Duplock, M. Scheffler, and P. J. D. Lindan, Phys. Rev. Lett. 92, 225502 (2004).

${ }^{38}$ M. Endo, T. Hayashi, S. H. Hong, T. Enoki, and M. S. Dresselhaus, J. Appl. Phys. 90, 5670 (2001).

${ }^{39}$ E. Hernandez, P. Ordejon, I. Boustani, A. Rubio, and J. A. Alonso, J. Chem. Phys. 113, 3814 (2000).

${ }^{40}$ T. B. Martins, R. H. Miwa, A. J. R. da Silva, and A. Fazzio, Phys. Rev. Lett. 98, 196803 (2007).

${ }^{41}$ A. Rycerz, J. Tworzydlo, and C. W. J. Beenakker, Nat. Phys. 3, 172 (2007).

${ }^{42}$ F. Munoz-Rojas, D. Jacob, J. Fernandez-Rossier, and J. J. Palacios, Phys. Rev. B 74, 195417 (2006).

${ }^{43}$ L. Brey and H. A. Fertig, Phys. Rev. B 73, 235411 (2006).

${ }^{44}$ Y. Son, M. L. Cohen, and S. G. Louie, Nature (London) 444, 347 (2006).

${ }^{45}$ J. Guo, D. Gunlycke, and C. T. White, Appl. Phys. Lett. 92, 163109 (2008).

${ }^{46}$ W. L. Wang, S. Meng, and E. Kaxiras, Nano Lett. 8, 241 (2008).

${ }^{47}$ P. Shemella, Y. Zhang, M. Mailman, P. M. Ajayan, and S. K. Nayak, Appl. Phys. Lett. 91, 042101 (2007).
${ }^{48}$ M. Ezawa, Phys. Rev. B 76, 245415 (2007).

${ }^{49}$ J. Fernandez-Rossier and J. J. Palacios, Phys. Rev. Lett. 99, 177204 (2007).

${ }^{50}$ D. Jiang, B. G. Sumpter, and S. Dai, J. Chem. Phys. 127, 124703 (2007).

${ }^{51}$ O. Hod, J. E. Peralta, and G. E. Scuseria, Phys. Rev. B 76, 233401 (2007).

${ }^{52}$ O. Hod, V. Barone, and G. E. Scuseria, Phys. Rev. B 77, 035411 (2008).

${ }^{53}$ E. Rudberg, P. Salek, and Y. Luo, Nano Lett. 7, 2211 (2007).

${ }^{54}$ T. J. Booth, P. Blake, R. R. Nair, D. Jiang, E. W. Hill, U. Bangert, A. Bleloch, M. Gass, K. S. Novoselov, M. I. Katsnelson, and A. K. Geim, Nano Lett. 8, 2442 (2008).

${ }^{55}$ W. Kohn and L. J. Sham, Phys. Rev. 140, A1133 (1965); P. Hohenberg and W. Kohn, ibid. 136, B864 (1964).

${ }^{56}$ J. P. Perdew, K. Burke, and M. Ernzerhof, Phys. Rev. Lett. 77, 3865 (1996).

${ }^{57}$ Developed by Atomistix Company, Copenhagen, Denmark, http://www.atomistix.com

${ }^{58}$ E. Durgun, S. Dag, V. M. K. Bagci, O. Gulseren, T. Yildirim, and S. Ciraci, Phys. Rev. B 67, 201401(R) (2003).

${ }^{59}$ H. J. Monkhorst and J. D. Pack, Phys. Rev. B 13, 5188 (1976).

${ }^{60}$ S. Tongay, R. T. Senger, S. Dag, and S. Ciraci, Phys. Rev. Lett. 93, 136404 (2004).

${ }^{61}$ J. A. Fürst, M. Brandbyge, A.-P. Jauho, and K. Stokbro, Phys. Rev. B 78, 195405 (2008).

${ }^{62}$ H. Xu and W. Sheng, Phys. Rev. B 57, 11903 (1998). 